\section{Creating Precise GFP Fusions in Plasmids Using Yeast Homologous Recombination}

BioTechniques 34:74-80 (January 2003)

\section{ABSTRACT}

Using a combination of primer amplification, homologous recombination, and yeast genetics, we established a method for creating precise promoter and protein fusions in genes originating from organisms other than yeast. One major advantage of this new method is its versatility. Fusions can be produced within a target gene without constraints regarding the site of insertion. Thus, fusions can be generated within a target sequence exactly at the site desired, and all sequences upstream and downstream of the insertion site were preserved. To illustrate the general applicability of this technique, we fused the gene encoding GFP to a Caenorhabditis elegans homologue of the dishevelled gene, dsh-2. This approach is not restricted to GFP fusions but can be utilized to create fusions between almost any two sequences regardless of the source. Therefore, this method provides a flexible alternative to other PCR-mediated techniques.

\section{INTRODUCTION}

In Caenorhabditis elegans, and many other organisms, GFP fusions are widely used as reporters of gene expression and protein localization. PCRbased strategies have been effectively utilized where coding sequences and $5^{\prime}$ regulatory sequences are amplified from genomic DNA and subcloned upstream of GFP. Because fusion of GFP is restricted to the $\mathrm{C}$-terminus of the targeted protein, endogenous $3^{\prime}$ untranslated regions (UTRs) and potential regulatory elements downstream of the gene are absent. Indeed, many $C$. elegans genes have 3' UTRs that are important in their translational regulation (7). For these genes, GFP fusions made by PCR amplification are unlikely to reflect the pattern of endogenous protein expression. An alternative method to ensure the presence of all the necessary regula- tory sequences is to insert GFP into a genomic subclone containing sequences extending both upstream and downstream of the gene of interest. However, the insertion of GFP is dependent on the availability of unique restriction sites within the cloned sequence, or an additional step is required to engineer a site. Recently, other methods have been devised where a gene of interest and GFP are fused through PCR amplification without the need for subcloning $(8,9)$. This particular method extends the utility of PCR such that GFP can be inserted within genes (8).

We have devised a method to insert GFP at any position within a subcloned sequence by using yeast homologous recombination. Although this method does utilize PCR amplification, problems inherent to most PCR-based methods are avoided by this recombinational fusion technique. To demonstrate the utility of this approach, we inserted GFP into different target sites within in a genomic construct containing the $C$. elegans dishevelled homologue, $d s h-2$. Dishevelled is a key component in two conserved intracellular signaling pathways, the Wnt signaling pathway and the planar cell polarity pathway (4). Based on other successful protein fusions made with dishevelled family homologues (2), we fused GFP to the C-terminus of DSH-2 leaving the endogenous $3^{\prime}$ UTR and downstream sequences intact. We also created a transcriptional fusion by inserting GFP at the initiating ATG codon. All sequences downstream of the insertion site were maintained. This approach is not limited to creating GFP fusions with $C$. elegans genes but can be exploited to create fusions with genes from any organism.

\section{MATERIALS AND METHODS}

\section{Yeast Strains and Media}

Yeast culture media, including YPD-rich medium and supplemented yeast minimal (YM) synthetic medium, were as described previously (1). Cycloheximide-resistant yeast were selected on YM solid medium containing $3 \mathrm{mg} / \mathrm{L}$ cycloheximide. The yeast strains constructed and described in this paper are CBS17 (MAT $\alpha$ his $3 \Delta 200$ leu2 $\Delta 1$ ura3-52 mal hap2) and CBY668 (MATo ade2-101 leu2-3,112 ura3-52 cyh2 kar1-1).

\section{Cloning and Plasmid Constructions}

Restriction enzymes used for cloning were purchased from New England Biolabs (Beverly, MA, USA), and cloning techniques were performed as described previously (11).

A genomic clone containing the $C$. elegans dsh homologue C27A2.6 was constructed by subcloning an approximately $19-\mathrm{kb} A v r \mathrm{II} / P s t \mathrm{I}$ fragment from cosmid K10B6 into the $X b a \mathrm{I} / P$ st $\mathrm{I}$ sites of pRS415, a LEU2-marked CEN/ARS plasmid (12), creating the plasmid pNH42. This clone contained two predicted genes upstream of C27A2.6, both of which were removed by digesting pNH42 with StuI and EagI and rejoining the ends of the plasmid, thereby generating pNH43. pNH43 contains 779 bp upstream of the C27A2.6 translational start site and $4 \mathrm{~kb}$ downstream of the stop codon. pNH44, which contains $5 \mathrm{~kb}$ of sequence upstream of C27A2.6, was generated by digesting pNH42 with $\mathrm{Sac}$ I to remove a $6.8-\mathrm{kb}$ fragment, followed by re-ligation of the plasmid.

The plasmid pCB182 (Figure 1) was constructed by inserting three fragments into the $S a c \mathrm{I} / E c o$ RI sites of pBluescript ${ }^{\circledR} \mathrm{KS}(+)$ (Stratagene, La Jolla, CA, USA), two of which contain direct repeats of GFP (GFP-S65Y) that flank on either side a $1.2-\mathrm{kb} P v u \mathrm{II} /$ SmaI fragment containing URA3 (Figure 1). The 679-bp SacI/PvuII GFP fragment upstream of $U R A 3$ was subcloned from pJR1862 (13), and the downstream GFP repeat was a 546-bp $M s c \mathrm{I} / E c o$ RI fragment derived from the same plasmid.

Amplification of the GFP-URA3GFP cassette from pCB182 was performed using Taq Precision Plus, with the buffer and primer concentrations used as described by the manufacturer (Stratagene). The amplification conditions used an annealing temperature of $45^{\circ} \mathrm{C}$, an extension time of $3 \mathrm{~min}$ at $72^{\circ} \mathrm{C}$, for 35 cycles. As described in the Results section, two amplified products were produced. The larger fragment was the desired product and did not require purification before transformation. 


\section{Microbial and Genetic Techniques}

Transformations into yeast were performed by the lithium acetate method (6). Plasmids were recovered from yeast as previously described (1) and transformed into E. coli DH5 $\alpha$ by electroporation. Yeast transformants containing pNH43 or pNH44 were selected on YM solid medium containing histidine and uracil (but lacking leucine). Transformants containing the GFP-URA3-GFP PCR fragment were selected on YM solid media supplemented with histidine and lacking both uracil (to select for cells containing the PCR fragment) and leucine (to maintain the presence of the LEU2-marked plasmid). All transformants were restreaked to single colonies on the appropriate media.

To transfer GFP-URA3-GFP-containing plasmids by yeast mating, exceptional cytoductants were selected after CBS17 donor strains were mated with the karyogamy-deficient karl-1 recipient strain, CBY668. Before mating, the CBS17 donor strains had been streaked as patches on YM solid medium supplemented with histidine and grown overnight at $30^{\circ} \mathrm{C}$. To facilitate mating between strains, the karl-1 strain was grown overnight at $30^{\circ} \mathrm{C}$ as a lawn on solid YPD-rich medium and replica-printed onto a fresh plate containing YPD-rich medium. The donor strain patches were then replica-printed on top of the karl-1 recipient. The strains were mated at $30^{\circ} \mathrm{C}$ overnight, and plasmid cytoductants were selected on YM solid medium containing adenine, uracil, and cycloheximide. Redcolored papillae were streaked on the

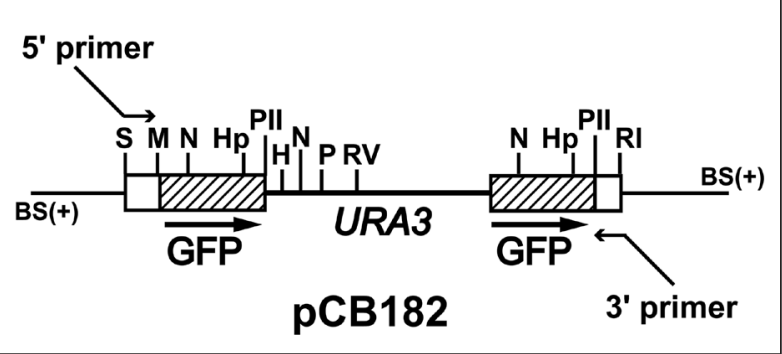

Figure 1. Plasmid map of amplification template pCB182. The plasmid pCB1 82 contained two tandem repeats of GFP (hatched boxes) that flank URA3. Primers chosen included sequences complementary to unique regions of the plasmid (white boxes) that permitted amplification of the GFP-URA3-GFP cassette. H, HindIII; Hp, HpaI; M, MscI; N, NdeI; P, PstI; PII, PvuII; RI, EcoRI; RV, EcoRV; S, SacI. same medium to isolate single colonies. The single colonies were then streaked as patches onto YM solid medium containing adenine and uracil and replicaprinted onto YM solid medium containing only adenine.

To select yeast in which homologous recombination had removed the intervening URA3 gene between the GFP repeats, karl-1 plasmid cytoductants were streaked onto YM solid medium supplemented with both adenine and uracil and grown overnight at $30^{\circ} \mathrm{C}$. These strains were replica-printed onto YM solid medium containing adenine, uracil, and 5-fluoroorotic acid (Toronto Research Chemicals, Toronto, ON, Canada) (3). Papillae were streaked to single colonies and were then inoculated into YM liquid medium supplemented with adenine and uracil for the isolation of the final GFP fusion plasmid from yeast. The plasmids were then transformed into $E$. coli from which the plasmid DNA was isolated. The insertion of GFP was confirmed by restriction analysis and sequencing.

\section{RESULTS}

\section{Amplification of the GFP-URA3- GFP Cassette}

The first step in this GFP fusion method was the amplification of a DNA fragment containing two direct repeats of GFP. The template for amplification was the plasmid pCB182, which contained the yeast $U R A 3$ gene flanked on either end by of GFP (Figures 1 and 2A). Each GFP repeat encoded most but not all of GFP. The GFP unit upstream of URA3 had a $5^{\prime}$ sequence extension not present in the downstream repeat, while the downstream repeat had a $3^{\prime}$ sequence extension not found in the upstream GFP unit. Sequences complementary to each unique extension (Table 1) were incorporated into each of the oligonucleotide primer sequences required to amplify the entire
GFP-URA3-GFP fragment (Figure 2A). Added onto the $5^{\prime}$ ends of the core primer sequences were at least $50 \mathrm{bp}$ of sequence complementary to the target DNA. These additional 50-bp sequences were chosen to produce the desired fusion junctions between GFP and the target DNA and to direct the location of homologous recombination. For the targeted insertion of GFP at the $3^{\prime}$ end of $d s h-2$ structural gene, the $5^{\prime}$ primer contained 51 nucleotides complementary to sequences immediately upstream of the $d s h-2$ stop codon (DSH42 primer; Table 1). The gene specific sequence for the 3' primer contained the stop codon followed by an additional 48 nucleotides (DSH43 primer; Table 1). The primers DSH59 and DSH60 (Table 1) were used to target insertion of GFP immediately downstream of the initiating $d s h-2$ ATG codon. A frameshift was incorporated into the $3^{\prime}$ primer (DSH60) to prevent

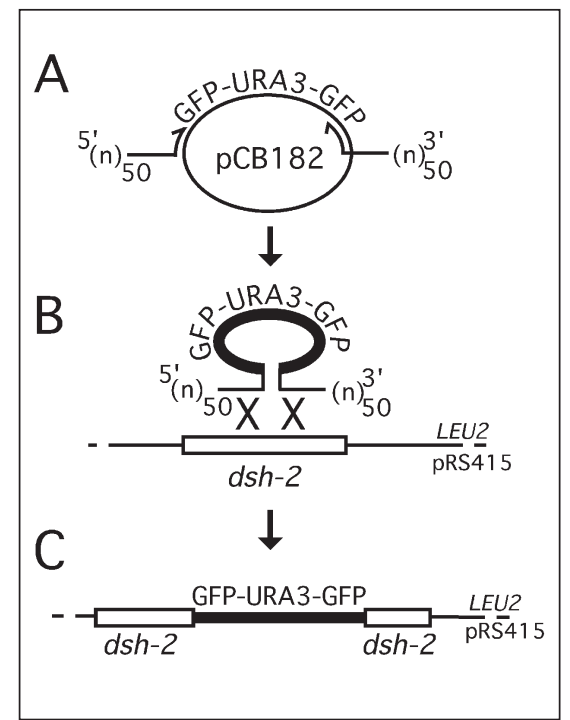

Figure 2. Strategy for the precise insertion of GFP into target plasmids. (A) Using the template plasmid pCB182 and primers in which complementary core sequences are incorporated (see Table 1), a fragment is amplified that contains the GFP-URA3-GFP DNA cassette. At the ends of the primers, approximately 50-bp sequences are added that are complementary to the target DNA within which GFP will be inserted. (B) After transformation into yeast, homologous recombination is directed by the approximately 50-bp extensions incorporated into the ends of the amplified PCR product into the target sequence (e.g., $d s h$-2) on a $L E U 2$-marked plasmid. (C) Yeast recombinants that carry the GFPURA3-GFP cassette are selected on solid medium lacking uracil and leucine (see Microbial and Genetic Techniques section). 
the fusion of GFP to the $d s h-2$ open reading frame. The PCR amplification produced two predicted fragments. Initial rounds of amplification produced a larger fragment (2.3-2.4 kb) containing both GFP repeats flanking URA3. However, after the first rounds, amplified products can themselves anneal together, thereby permitting the production of a smaller approximately 800-bp fragment, which contained only one of the GFP repeats and lacked the URA3 gene. The amplification of the smaller undesired fragment was slightly favored but purification of the full-length product before transformation into yeast was not necessary. Because the smaller amplification product did not contain the URA3 gene, yeast transformants carrying this fragment did not propagate on the selective medium used.

\section{Insertion of the GFP-URA3-GFP Cassette into the Target Sequence}

As the first step towards creating a GFP/DSH-2 protein fusion, the GFP$U R A 3-$ GFP product generated with the DSH42/43 primers was transformed into the yeast strain CBS17 containing the plasmid pNH44. A DSH-2/GFP transcriptional fusion was produced using the product amplified with the DSH59/60 primers, and it was transformed into $\mathrm{CBS} 17$ containing the plasmid pNH43 (Figure 2, B and C). Both pNH43 and pNH44 are genomic clones containing the entire $d s h-2$ gene, downstream flanking sequences, and differing amounts of upstream sequence, all subcloned into the yeastbacterial shuttle vector pRS415. Cells bearing plasmid subclones into which the amplified GFP-URA3-GFP product had been inserted were selected on solid medium lacking uracil (to select for the amplified product) and leucine (to select for the plasmid). A total of 11 transformants were isolated for the DSH-2/GFP protein fusion, and three transformants were isolated for the transcriptional fusion.

\section{Selection of Strains Carrying a Homogeneous Population of GFP- URA3-GFP-Containing Plasmids}

The GFP-URA3-GFP PCR product can integrate by homologous recombi-

Table 1. Sequences of Amplification Primers

\begin{tabular}{|ll|}
\hline Oligonucleotide Primer & Sequence $^{\mathbf{a}}$ \\
\hline Core upstream primer sequence: & $5^{\prime}$-atgagtaaaggagaagaacttttcac-3' \\
Core downstream primer sequence: & $5^{\prime}$-gaattctttgtatagttcatccatgc-3' \\
dsh-2 upstream primer, DSH42 & $5^{\prime}$-TCGTTTCGAGCTGCAATGAACG- \\
(translational fusion): & GAAGTCATCCACAGTTTAACATTGA- \\
& TAATatgagtaaaggagaagaactttcac-3' \\
dsh-2 downstream primer, DSH43 & $5^{\prime}$-ACAAATTAAAACATATCAAAAAATA- \\
(translational fusion): & CAGAGAAACAAGAACATCTACTAATCA- \\
& gaattcttgtatagttcatccatgc-3' \\
dsh-2 upstream primer, DSH59 & $5^{\prime}$-CTAGCTTAAAAGTATACTATTTTA- \\
(transcriptional fusion): & TTGTTTTTTTTCTTTTCAGATAAGA- \\
& CAAAatgagtaaaggagaagaacttttcac-3' \\
dsh-2 downstream primer, DSH60 & $5^{\prime}$-GTGGCAACATCTGATGCGTCAAA- \\
(transcriptional fusion): & TGATGAATCAATTGGTGAAGGGGAAT- \\
& CTGTCgaattcttgtatagttcatccatgc-3'
\end{tabular}

aCore sequences with homology to GFP are shown in lowercase bold lettering.

nation into the plasmid DNA or nonspecifically into the yeast genome. In addition, since yeast carry several copies of CEN-based plasmids, yeast transformants containing a GFPURA3-GFP PCR product that has recombined into one plasmid will also contain other plasmids that do not contain the amplified fragment. Yeast must be isolated that do not contain undesired plasmids that lack the GFP$U R A 3-$ GFP cassette, or they will interfere with the selection of the final GFP fusion. We used a simple technique that only required replica-printing yeast between different solid media to obtain GFP-URA3-GFP plasmids and the final GFP fusion construct. To create strains with a homogeneous population of plasmids containing the integrated GFP-URA3-GFP fragment and to select against undesired DNA insertions into chromosomal sites, individual plasmids were transferred into a karyogamy-deficient kar 1-1 recipient yeast strain by exceptional cytoduction (Figure 3A). Karyogamy is the process during yeast mating when haploid nuclei fuse and generate a diploid nucleus. The karl-1 mutation disrupts karyogamy, thereby limiting the exchange of chromosomal DNA between cells. However, the transfer of plasmids and extranuclear material can occur in karl-1 mutants $(5,10)$. Therefore, the karyogamy defect of kar 1-1 allows the selection of haploid cells that obtain plasmids and cytoplasm from a mating partner but retain their original nuclear genotype. To transfer plasmids, transformants carrying the GFP-URA3-GFP cassette were mated en masse with a karl-1 recipient yeast strain (CBY668) that was spread out as a lawn on solid rich medium. After mating on rich medium, kar1-1 recipients, into which the $d s h-2$ containing plasmids had been transferred, were selected on solid medium containing cycloheximide but lacking leucine. The cycloheximide resistance of the karl-1 strain (conferred by the cyh 2 mutation) is recessive; therefore, diploids and the original transformants did not grow in the presence of cycloheximide. The karl1 strain is also a leucine auxotroph and, therefore, will only grow on leucine-deficient medium if it received the plasmid from the CBS17 strain. We then tested the karl-1 recipient strains for the presence of $U R A 3$, indicating that the GFP-URA3-GFP cassette was also transferred and thus integrated into the plasmid (see Microbial and Genetic Techniques section). For the GFP/DSH-2 protein fusion, the GFP-URA3-GFP cassette 
was plasmid-linked in three of the 11 transformants. These three plasmids were isolated from yeast, transformed into E. coli, and plasmid DNA was prepared. Two of the purified plasmids contained the GFP-URA3-GFP fragment correctly inserted at the $3^{\prime}$ end of $d s h-2$. For the transcriptional fusion, two of the three insertion events were plasmid-linked.

\section{Generation of the Final GFP Fusion Construct}

The final step was the selection of cells that "loop-out" URA3 by recombination between the two homologous GFP repeats, thus creating an intact
GFP open reading frame within the targeted sequence (Figure 3B). Cells were grown on solid medium lacking leucine (which selected for the plasmid) and containing 5-fluoroorotic acid, which selected against cells containing the $U R A 3$ gene. After the recovery of these GFP-fusion plasmids from yeast, the final constructs were verified by restriction mapping and sequencing.

We discovered that the cytoduction step, performed before culturing yeast on 5-fluoroorotic acid, was essential to obtain plasmid recombinants. After the GFP-URA3-GFP had integrated into the target plasmid, the yeast cells also contained copies of the original plasmid that did not carry the integrated fragment. Growth in the presence of 5-fluoroorotic acid selects either for yeast in which the $U R A 3$ gene is lost by recombination or for yeast in which the plasmid containing the GFPURA3-GFP is lost during mitosis. The latter case was the most prevalent, and our attempts to directly obtain a $d s h-2::$ GFP fusion plasmid were only successful when the cytoduction step was implemented.

\section{DISCUSSION}

We have devised a method that takes advantage of homologous recombination in yeast to insert GFP at any position within a target sequence. Recently, a PCR-based method has been described that can also be used to insert GFP at any site within a sequence (8). This method is both rapid and simple; fusion constructs can be quickly generated, producing a linear product that does not require subcloning. Our method, however, offers a flexible alternative. One significant advan- tage is the generation of a subclone that can be subjected to further manipulations. In addition, there is less possibility for PCR-induced errors in the final product. Although our method also utilizes PCR, only GFP is amplified and any nucleotide errors can be easily detected by a single sequencing reaction. In our method, there are also fewer constraints with respect to the size of the gene targeted for GFP insertion. We have successfully fused GFP to the $C$. elegans gene C07G1.4 in the context of a large 18-kb genomic fragment. Thus, this method is suitable for GFP insertions into long sequences, and GFP can potentially be inserted into even larger subclones.

The methodology that we have described has applications beyond the examples presented here. Deletions can be generated either immediately upstream or downstream of the GFP insertion site by designing primers that recombine with the target DNA at noncontiguous sequences. Although the fusions created here had no extraneous sequence other than GFP itself, restriction sites or sequences encoding additional amino acids could be incorporated into the primers and thereby added to the final GFP fusion. Moreover, this methodology can be applied to DNA from any other organism once cloned into the appropriate yeast shuttle vector. Fusions other than those involving GFP can also be created. Additional vectors for amplification can be constructed in which other common protein fusion tags (e.g., GST, $\beta$-galactosidase, or maltose-binding protein) are used, instead of GFP, to make fusions. Thus, theoretically, one could insert almost any DNA sequence into any other target sequence.

\section{REFERENCES}

1.Adams, A., D.E. Gottschling, C.A. Kaiser, and T. Stearns. 1997. Methods in Yeast Genetics. CSH Laboratory Press, Cold Spring Harbor, NY.

2.Axelrod, J.D. 2001. Unipolar membrane association of Dishevelled mediates Frizzled planar cell polarity signaling. Genes Dev. 15:1182-1187.

3.Boeke, J.D., J. Trueheart, G. Natsoulis, and G.R. Fink. 1987. 5-fluoroorotic acid as a selective agent in yeast molecular genetics. Methods Enzymol. 154:164-175. 


\section{Short Technical Reports}

4.Boutros, M. and M. Mlodzik. 1999. Dishevelled: at the crossroads of divergent intracellular signaling pathways. Mech. Dev. 83:27-37.

5.Dutcher, S.K. 1981. Internuclear transfer of genetic information in karl-1/KARl heterokaryons in Saccharomyces cerevisiae. Mol. Cell. Biol. 1:246-253.

6.Gietz, R.D. and R.H. Schiestl. 1995. Transforming yeast with DNA. Methods Mol. Cell. Biol. 5:255-269.

7.Goodwin, E.B. and T.C. Evans. 1997. Translational control of development in C. elegans. Semin. Cell Dev. Biol. 8:551-559.

8.Hobert, O. 2002. PCR fusion-based approach to create reporter gene constructs for expression analysis in transgenic $C$. elegans. BioTechniques 32:728-730

9.Hobert, O., D.G. Moerman, K.A. Clark, M.C. Beckerle, and G. Ruvkun. 1999. A conserved LIM protein that affects muscular adherens junction and integrity and mechanosensory function in C. elegans. J. Cell Biol. 144:45-57.

10.Rose, M.D. 1996. Nuclear fusion in the yeast Saccharomyces cerevisiae. Annu. Rev. Cell. Dev. Biol. 12:663-695.

11.Sambrook, J., E.F. Fritsch, and T. Maniatis. 1989. Molecular Cloning, 2nd ed. CSH Laboratory Press, Cold Spring Harbor, NY.

12.Sikorski, R. and P. Hieter. 1989. A system of shuttle vectors and yeast host strains designed for efficient manipulation of DNA in Saccharomyces cerevisiae. Genetics 122:1927.

13.Whistler, J.L. and J. Rine. 1997. Ras2 and Ras1 protein phosphorylation in Saccharomyces cerevisiae. J. Biol. Chem. 272:18790-18800

We thank Qun Shan for his comments on the manuscript. This work was supported by a Research Project grant (RPG-98-068-01$D D C$ ) from the American Cancer Society and a National Institutes of Health grant (NS42213) to G.G. N.H. was supported by an American Heart Association postdoctoral fellowship, and C.T.B. was supported by a Leukemia and Lymphoma Society Special Fellows grant no. 3042-00. Address correspondence to Dr. Christopher T. Beh, Department of Molecular Biology and Biochemistry, Simon Fraser University, 8888 University Drive, Burnaby, B.C., Canada V5A 1S6.e-mail: ctbeh@sfu.ca
Received 21 June 2002; accepted 16 October 2002

\author{
Nancy C. Hawkins ${ }^{1,2}$, Gian \\ Garriga $^{2}$, and Christopher \\ T. Beh ${ }^{1,2}$ \\ ${ }^{1}$ Simon Fraser University \\ Burnaby, B.C., Canada \\ ${ }^{2}$ University of California \\ Berkeley, CA, USA
}

\section{For reprints of this or any other article, contact Reprints@BioTechniques.com}

